\title{
CONTRIBUTION A L'ÉTUDE DE L'ACTION DE LA CHALEUR SUR LES CASÉINES

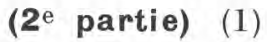 \\ par
}

Mme M. SWIRSKI, R. ALLOUF et H. CHEFTEL Laboratoire de Recherches des Etablissements J.-J. Carnaud et Forges de Basse-Indre, Billancourt (Seine)

Dans un travail antérieur [1] nous avons étudié l'influence du chauffage sur l' $\alpha$-caséine et la $\beta$-caséine en solution à $p H 7,2$. Les caractères suivants : couleur, indice de solubilité (apprécié par néphélométrie) entre $p H \mathbf{H}$ et 4 , constante de sédimentation, teneur en phosphore dialysable, allure de l'hydrolyse trypsique, sont modifiés par le chauffage $\left(40 \mathrm{mn}\right.$ à $\left.120^{\circ} \mathrm{C}\right)$ dans le cas de l' $\alpha$-caséine. Ils demeurent inchangés pour la $\beta$-caséine.

Dans le présent travail, nous nous sommes proposés :

10 D'étendre l'étude de l' $\alpha$-caséine :

a) D'une part à l'influence du chauffage sur certains autres caractères : solubilité à $p \mathrm{H} 4,5$, teneur en "lysine disponible » $[2,3]$, teneur en acides aminés possédant des radicaux $\mathrm{NH}_{2}$ libres $[4,5$, $6,7]$;

b) D'autre part, à la comparaison entre l' $\alpha$-caséine déphosphorylée par chauffage et l' $\alpha$-caséine déphosphorylée par l'action d'une phosphatase : on pouvait en effet se demander si la déphosphorylation de la caséine pouvait, à elle seule, rendre compte des modifications entraînées par le traitement thermique; dans ce cas les caractères étudiés ont été : indice de solubilité entre $p \mathrm{H} 7$ et 4 , allure de l'hydrolyse trypsique.

$2^{\circ}$ D'appliquer au lait divers traitements thermiques industriels afin d'examiner leur influence sur les caséines.

\section{Méthodes expérimentales}

\section{A) Préparation des caséines}

L' $\alpha$-caséine a été préparée selon la méthode de WARNER [8] à partir de lait (2) de vache écrémé préalablement.

(1) Bull. Soc. Ch. biol., 1963, 45, 901.

(2) Le lait utilisé dans cette étude nous a été procuré par M. J. Pien, Directeur des Laboratoires de la Soeiété des Fermiers Réunis, auquel nous tenons à renouveler ici nos très vifs remerciements. 
La déphosphorylation de l'a-caséine a été effectuée, selon la méthode décrite par KeLLeY [9], à l'aide de phosphatase intestinale (1). La dose de phosphatase mise en œuvre a été de $400 \mathrm{mg} / \mathrm{l}$ de solution protéique à 2 p. 100. L'arrêt de la déphosphorylation a été obtenu par dialyse du mélange protéine-enzyme contre tampon borate de $p H$ 7,2 ( $/ 2=0,118)[10]$. L'absence d'action protéolytique de la phosphatase sur l' $\alpha$-caséine dans les conditions de nos expériences a été vérifiée.

La deuxième partie de ce travail a été effectuée à l'aide de lait entier de vache. Ce lait a été, selon les cas, chauffé ou non chauffé ; puis les caséines ont été séparées comme suit :

- refroidissement du lait à $4^{\circ} \mathrm{C}$;

- centrifugation à $4^{\circ} \mathrm{C}$ pendant $90 \mathrm{mn}(1380 \times \mathrm{g})$;

- dialyse pendant 48 heures à $4^{\circ} \mathrm{C}$ contre tampon phosphate $(\Gamma / 2=0,118)$ ou borate $(\Gamma / 2=1,14)$ de $p H ~ 7,2$.

\section{B) Méthodes analytiques}

Outre les techniques mises en œuvre au cours de notre précédente étude sur les caséines [1], nous avons appliqué les méthodes suivantes :

\section{Azote $\alpha$-aminé.}

Le dosage de l'azote $\alpha$-aminé a été effectué manométriquement selon la méthode de VAN SLYKE et coll. [11, 12] par réaction avec la ninhydrine.

\section{Solubilité à $p \mathrm{H}$ 4,5.}

La solubilité à $p H$ 4,5 de l' $\alpha$-caséine a été évaluée comme suit : une solution d' $\alpha$-caséine à 2 p. 100 dans du tampon phosphate de $p \mathrm{H} 7,2$ a été dialysée pendant 70 heures à $4^{\circ} \mathrm{C}$ contre un tampon acide citrique/phosphate de sodium de $p H 4,5(\Gamma / 2=0,35)$. Le contenu du sac à dialyse a été centrifugé à $4^{\circ} \mathrm{C}$ pendant 1 heure $(4240 \times \mathrm{g})$. L'azote contenu dans le surnageant ainsi obtenu, a été dosé. La teneur en protéine soluble a été déduite de l'azote en appliquant le coefficient 6,43 [13].

$3^{\circ}$ "Lysine disponible ".

En appliquant la méthode de CARPenter et coll. [2, 3] nous avons déterminé les radicaux $\mathrm{NH}_{2}$ non dissimulés vis-à-vis du 
dinitrofluorobenzène. L'intérêt de cette détermination réside dans l'hypothèse de CARPENTER, selon laquelle les radicaux dissimulés vis-à-vis du dinitrofluorobenzène le sont également vis-à-vis des enzymes protéolytiques du tractus gastro-intestinal. Etant donné que, parmi les radicaux $\mathrm{NH}_{2}$, les radicaux $\varepsilon-\mathrm{NH}_{2}$ sont les plus nombreux, cette mesure permet d'évaluer ce que CARPENTER et coll. ont appelé "lysine disponible», et de juger l'influence des traitements industriels sur la "disponibilité " de la lysine présente dans les produits alimentaires.

\section{$4^{\circ}$ Acides aminés présentant des radicaux $\mathrm{NH}_{2}$ libres.}

Afin de préciser les résultats des déterminations de la "lysine disponible ", les acides aminés possédant des radicaux $\mathrm{NH}_{2}$ libres ont été isolés puis dosés en appliquant la méthode au fluorodinitrobenzène décrite par SANGER [14] et la technique de séparation chromatographique de Monnier [15].

L'élution des spots correspondant aux acides aminés dinitrophénylés a été effectuée à l'aide d'une solution de bicarbonate de sodium à 1 p. $100 \mathrm{p} / \mathrm{v}$. Le dosage colorimétrique proprement dit a été fait au moyen d'un spectrophotomètre Beckman DU.

\section{C) Traitements thermiques industriels appliqués au lait}

Les différents traitements thermiques retenus pour cette étude ont été les suivants :

1. Pasteurisation pendant 20 à 30 secondes à $90^{\circ} \mathrm{C}(1)$;

2. Stérilisation en boîte métallique par passage rapide des récipients sur des flammes de propane (stérilisateur continu "Stériflamme " [16]) pour atteindre la température de $122^{\circ} \mathrm{C}$, maintien de cette température pendant 4 minutes, refroidissement par immersion de la boîte dans de la glace;

3. Stérilisation en boîte métallique dans un autoclave : montée en température en $16 \mathrm{mn}$, maintien à $118^{\circ} \mathrm{C}$ pendant $12 \mathrm{mn}$, refroidissement en $22 \mathrm{mn}$;

4. Stérilisation en bouteille dans un autoclave : montée en température en $31 \mathrm{mn}$, maintien à $118^{\circ} \mathrm{C}$ pendant $12 \mathrm{mn}$, refroidissement en $47 \mathrm{mn}$.

(1) Cette opération a été effectuée grâce à l'obligeance de M. Pien. 


\section{Résultats expérimentaux}

\section{$\mathrm{I}-\alpha$-Caséine}

10 Solubilité à $p \mathrm{H} \quad 4,5$ à $4^{\circ} \mathrm{C}$.

Les valeurs ci-après indiquent que le chauffage a pour effet de doubler pratiquement la solubilité de $1^{\prime} \alpha$-caséine à $p H 4,5$ à $4^{\circ} \mathrm{C}$.

$\mathrm{N} \alpha$-caséine non chauffée $\ldots \ldots \ldots \ldots \ldots \ldots, 0,33 \mathrm{~g} / \mathrm{l}$

$\mathrm{N} \alpha$-caséine chauffée $\left(40 \mathrm{mn}\right.$ à $\left.120^{\circ} \mathrm{C}\right) \ldots 0,61 \mathrm{~g} / \mathrm{l}$

\section{$2^{\circ}$ "Lysine disponible."}

Les résultats des déterminations de la "lysine disponible " sont consignés dans le tableau ci-dessous. Ils indiquent que le chauffage (traitement industriel de $12 \mathrm{mn}$ à $118^{\circ} \mathrm{C}$ ) a pour effet de "dissimuler " une proportion importante de la lysine vis-à-vis du fluorodinitrobenzène et ce, surtout dans le cas où la solution $d^{\prime} \alpha$-caséine est additionnée de lactose.

TABLEAU I

Solution à 2,6 p. 100 d' $\alpha$-caséine non chauffée .... Solution à 2,6 p. 100 d' $\alpha$-caséine chauffée .......

Solution à 2,6 p. 100 d' $\alpha$-caséine et à 4 p. 100 de

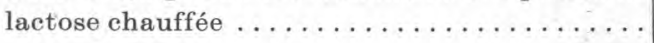

\begin{tabular}{|c|c} 
Lysine & \\
disponible & Perte \\
mg p. $100 \mathrm{~g}$ & p. 100 \\
d' $\alpha$-caséine & \\
\hline & \\
\hline 2,8 & 0 \\
2,5 & 12 \\
& \\
1,9 & 33
\end{tabular}

$3^{\circ}$ Acides aminés possédant des radicaux $\mathrm{NH}_{2}$ libres.

La détermination des radicaux $\mathrm{NH}_{2}$ libres de la lysine et de l'arginine dans l' $\alpha$-caséine avant et après un traitement thermique de $12 \mathrm{mn}$ à $118^{\circ} \mathrm{C}$, analogue à celui appliqué le plus souvent pour la stérilisation industrielle du lait, a fourni les valeurs suivantes (cf. tableau II).

Ces déterminations ont mis en évidence un "blocage " très étendu des groupes aminés libres sous l'influence du chauffage. L'addition de lactose a pour effet d'accroître ce blocage au point 
TABLEAU II

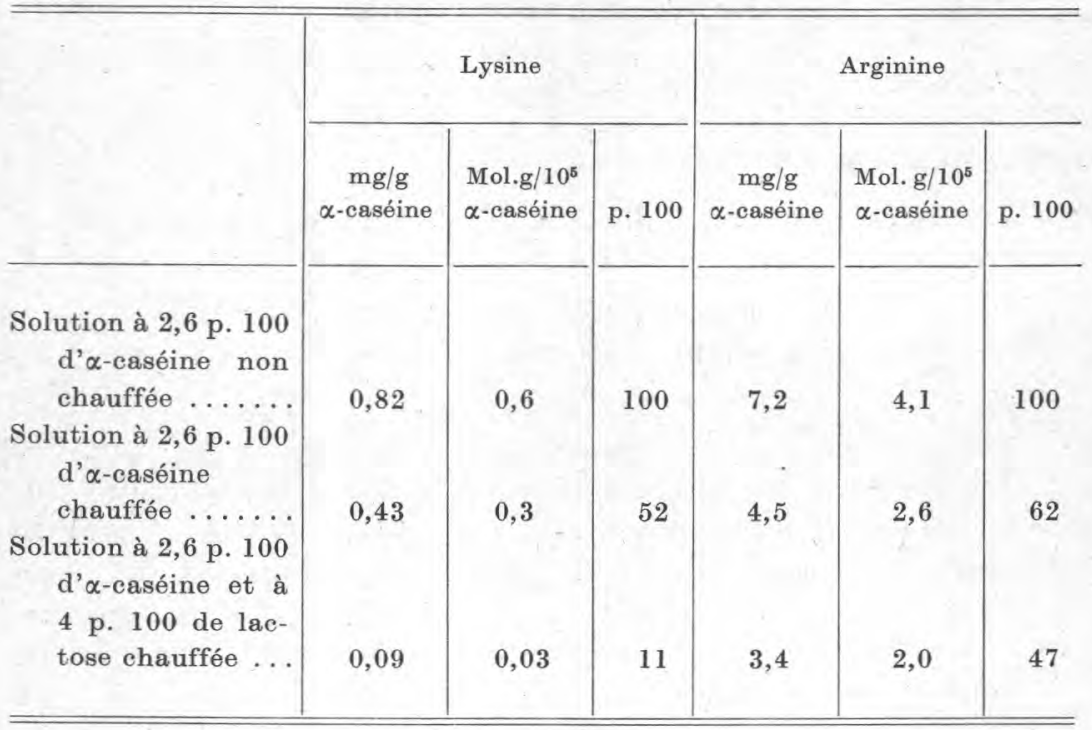

que les groupes aminés de la lysine sont, après chauffage, pratiquement tous dissimulés au fluorodinitrobenzène.

$4^{\circ} \alpha$-caséine déphosphorylée par voie enzymatique et a-caséine déphosphorylée par chauffage.

a) Indice de solubilité.

Les variations, en fonction du $p \mathrm{H}$, de l'indice de solubilité sont pratiquement identiques, qu'il s'agisse d' $\alpha$-caséine déphosphorylée à l'aide de phosphatase ou d' $\alpha$-caséine déphosphorylée par chauffage $\left(40 \mathrm{mn}\right.$ à $120^{\circ} \mathrm{C}$ ), la proportion de phosphore éliminé (environ 50 p. 100) étant la même dans les deux cas (fig. 1).

Ainsi l'élimination d'un certain nombre de groupes phosphoryle paraît être liée à ces modifications de solubilité.

b) Action de la trypsine.

Le chauffage de l' $\alpha$-caséine a pour effet d'augmenter sensiblement la résistance de celle-ci à la trypsine, mais la déphosphorylation ne paraît pas être la cause de cette modification. En effet, l' $\alpha$-caséine déphosphorylée par voie enzymatique, - donc sans faire appel à un traitement thermique, - est attaquée par la trypsine pratiquement à la même vitesse que l' $\alpha$-caséine non chauffée et non déphosphorylée (fig. 2). 


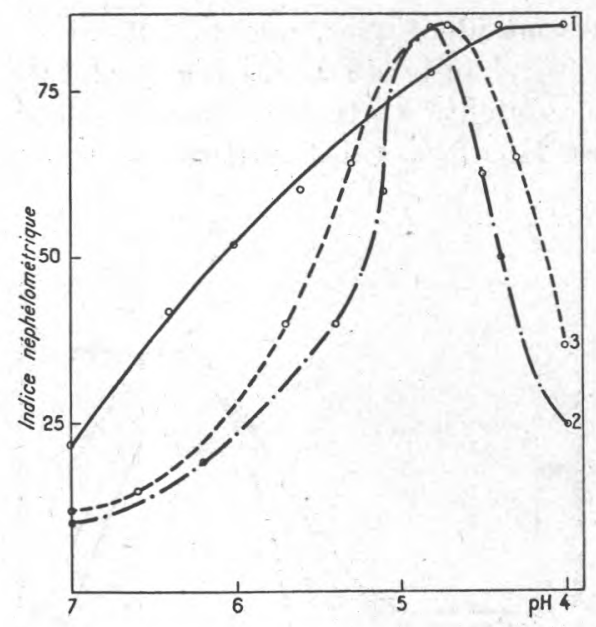

Fig. 1. - Indice de solubilité de l' $\alpha$-caséine en fonction du $p H$.

1. Native.

2. Chauffée.

3. Déphosphorylée enzymatiquement.

Ces résultats confirment ceux qui ont été obtenus avec la caséine totale par T. A. Sundararajan et coll. [17].

$$
\text { II - Lait }
$$

10 Indice de solubilité entre $p \mathrm{H} 7$ et $p \mathrm{H} 4$.

Les variations de l'indice de solubilité en fonction du $p \mathrm{H}$ des

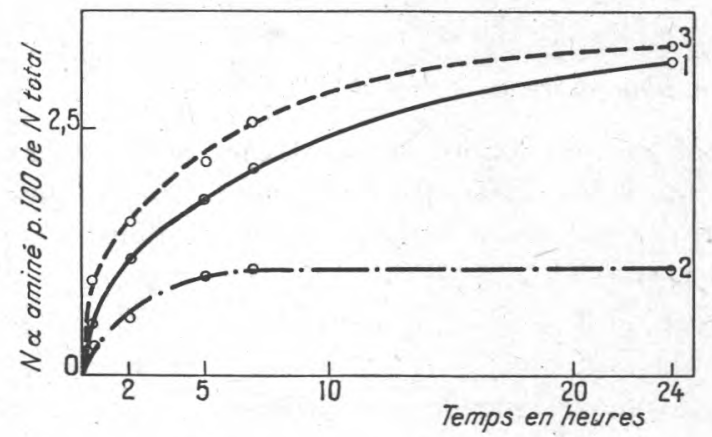

Fig. 2. - Hydrolyse trypsique de l' $\alpha$-caséine.

1. Native.

2. Chauffée.

3. Déphosphorylée enzymatiquement. 
caséines du lait sont identiques, que ce lait ait été pasteurisé ou qu'il n'ait pas été chauffé du tout. L'augmentation, du fait $d u$ chauffage, de la solubilité à $p \mathrm{H} 4$ se retrouve à des degrés plus ou moins prononcés dans le cas des traitements thermiques de stérilisation (fig. 3).

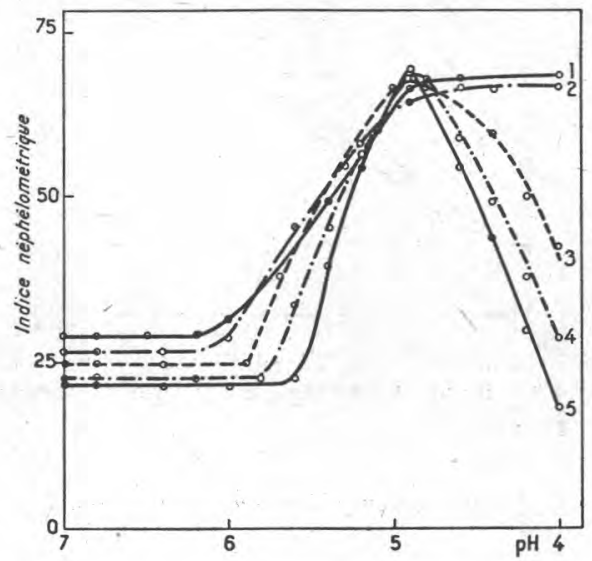

Fig. 3. - Indice de solubilité entre $p \mathrm{H} 7$ et 4 du lait soumis à différents traitements thermiques.

1. Cru.

2. Pasteurisé 30 sec à $90^{\circ} \mathrm{C}$.

3. Chauffé sur flamme $4 \mathrm{mn}$ à $122^{\circ} \mathrm{C}$.

4. Autoclavé (boîte) $12 \mathrm{mn}$ à $118^{\circ} \mathrm{C}$.

5. Autoclavé (bouteille) $12 \mathrm{mn}$ à $118^{\circ} \mathrm{C}$.

\section{$2^{\circ}$ Teneur en phosphore non dialysable.}

Les pertes en phosphore minéral, occasionnées aux caséines du lait à la suite des différents traitements thermiques appliqués, ont été déduites des teneurs résiduelles en phosphore non dialysable (cf. tableau III).

Les résultats ci-dessus indiquent que les traitements thermiques appliqués se classent dans le même ordre tant en ce qui concerne les pertes en phosphore que les modifications de solubilité qu'ils entraînent. Le rôle important des groupes phosphoryle dans la solubilité des caséines se trouve ainsi confirmé.

La pasteurisation et la stérilisation rapide des boîtes sur flamme n'entraînent que des pertes minimes en phosphore. 
TABLEAU III

\begin{tabular}{l|c|c}
\hline \hline \multicolumn{1}{c|}{ Lait } & $\begin{array}{c}\text { Mg P } \\
\text { non dialysable/ } \\
\text { litre lait }\end{array}$ & Perte p. 100 \\
\hline & & \\
Non chauffé $\ldots \ldots \ldots \ldots \ldots \ldots \ldots \ldots \ldots \ldots$ & 245 & 0 \\
Pasteurisé $\ldots \ldots \ldots \ldots \ldots \ldots \ldots \ldots \ldots \ldots$ & 210 & 14,3 \\
Stérilisé sur flamme $\left(4 \mathrm{mn}\right.$ à $\left.122^{\circ} \mathrm{C}\right) \ldots \ldots \ldots$ & 217 & 11,4 \\
Autoclavé en boîte $\left(12 \mathrm{mn}\right.$ à $\left.118^{\circ} \mathrm{C}\right) \ldots \ldots \ldots$ & 185 & 24,5 \\
Autoclavé en bouteille $\left(12 \mathrm{mn}\right.$ à $\left.118^{\circ} \mathrm{C}\right) \ldots$ & 148 & 39,8 \\
& & \\
\hline \hline
\end{tabular}

$3^{\circ}$ "Lysine disponible. "

Les résultats des déterminations de la "lysine disponible " sont consignés dans le tableau ci-dessous :

TABLEAU IV

\begin{tabular}{|c|c|c|}
\hline Lait & $\begin{array}{c}\text { Mg lysine } \\
\text { disponible/ } \\
\text { litre lait }\end{array}$ & Perte p. 100 \\
\hline Non chauffé & 2,70 & 0 \\
\hline Pasteurisé $\ldots \ldots \ldots \ldots \ldots \ldots \ldots \ldots$ & 2,62 & 3 \\
\hline Stérilisé en boîte $\left(4 \mathrm{mn}\right.$ à $\left.122^{\circ} \mathrm{C}\right) \ldots \ldots \ldots$ & 2,30 & 15 \\
\hline Stérilisé en bouteille ( $12 \mathrm{mn}$ à $\left.118^{\circ} \mathrm{C}\right) \ldots \ldots$ & 1,90 & 30 \\
\hline
\end{tabular}

$4^{\circ}$ Composition en acides aminés.

Bigwood [18] ayant signalé qu'un chauffage pendant 20 minutes à $122-124^{\circ} \mathrm{C}$ du lait écrémé entraînait la destruction d'environ 10 p. 100 de la lysine, nous avons dosé les acides aminés des caséines du lait après avoir fait subir à ce dernier divers traitements thermiques (cf. tableau V).

Compte tenu de la précision $( \pm 10 \mathrm{p}$. 100) de la méthode de dosage utilisée, nous n'avons pas pu mettre en évidence de variations significatives de la composition en acides aminés qui puissent être attribuées au traitement thermique.

Ce résultat confirme celui qui a déjà été obtenu pour la caséine totale au cours d'un travail précédent [1]. 
TABLEAU $V$

COMPOSITION EN ACIDES AMINÉS EN mg/litre DE LAIT

\begin{tabular}{|c|c|c|c|c|}
\hline an? , thetes & $\begin{array}{c}33^{3} \\
=,+3\end{array}$ & Cru & $\begin{array}{c}\text { Stérilisé } \\
\text { sur flamme }\end{array}$ & $\begin{array}{l}\text { Autoclavé } \\
\text { en bouteille }\end{array}$ \\
\hline Acide aspartique & $\ldots \ldots \ldots \ldots$ & 245 & 240 & 230 \\
\hline Acide glutamique & $\ldots \ldots \ldots$ & 720 & 715 & 680 \\
\hline Lysine ......... & $\ldots \ldots \ldots$ & 261 : & $260 \cdots$ & 246 \\
\hline Histidine $\ldots .$. & 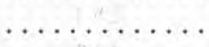 & 65 & 64 & 65 \\
\hline Arginine $\ldots . .$. & . A. . . & $121 *$ & $\therefore 117$ & to 118 \\
\hline Leucines . . . . . & $\ldots \ldots \ldots \ldots$ & 425 & 434 & 425 \\
\hline Glycine ....... & $\ldots \ldots \ldots$ & 26 & 26 & 29 \\
\hline Alanine $\ldots . .$. & $\ldots \ldots \ldots \ldots$ & 95 & 300 & $\therefore \quad 95$ \\
\hline Valine ....... & 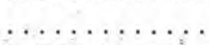 & 194 & 195 & 194 \\
\hline Proline . . . & $\ldots \ldots \ldots$ & 245 & - 240 & 245 \\
\hline Phénylalanine .. & $\ldots \ldots \ldots \ldots$ & 162 & 160 & 162 \\
\hline Cystine ........ & $\ldots \ldots \ldots \ldots$ & 14 & 12 & 12 \\
\hline Méthionine .... & $\ldots \ldots \ldots$ & A 3 , 94 & 96 & 92 \\
\hline Sérine ....... & $\ldots \ldots$ & 158 & 160 & 158 \\
\hline Thréonine ..... & & 165 & 168 & 165 \\
\hline Tyrosine $\ldots \ldots \cdots$ & & 136 & 135 & 136 \\
\hline
\end{tabular}

$5^{\circ}$ Action de la trypsine.

a) L'allure de l'hydrolyse trypsique des caséines du lait soumis à différents traitements thermiques industriels a été déterminée (fig. 4).

La trypsine attaque apparemment aussi aisément les caséines du lait non chauffé que celles du lait pasteurisé et du lait stérilisé en boîte pendant 4 minutes à $122^{\circ} \mathrm{C}$. Au contraire, l'action de la trypsine est sensiblement ralentie dans le cas des caséines provenant de lait stérilisé, en boîte ou en bouteille, pendant 12 minutes à $118^{\circ} \mathrm{C}$.

b) Les acides aminés libérés par l'action de la trypsine ont été dosés après divers temps de protéolyse.

En raison des difficultés de séparation dues à la présence de peptides, nous n'avons dosé que cinq des acides aminés libérés : acide aspartique, acide glutamique, histidine, lysine et arginine.

D'autre part, les acides aminés constituant la fraction non précipitable par l'acide perchlorique ont été dosés par hydrolyse acide. Les figures 5 et 6 résument nos résultats. 


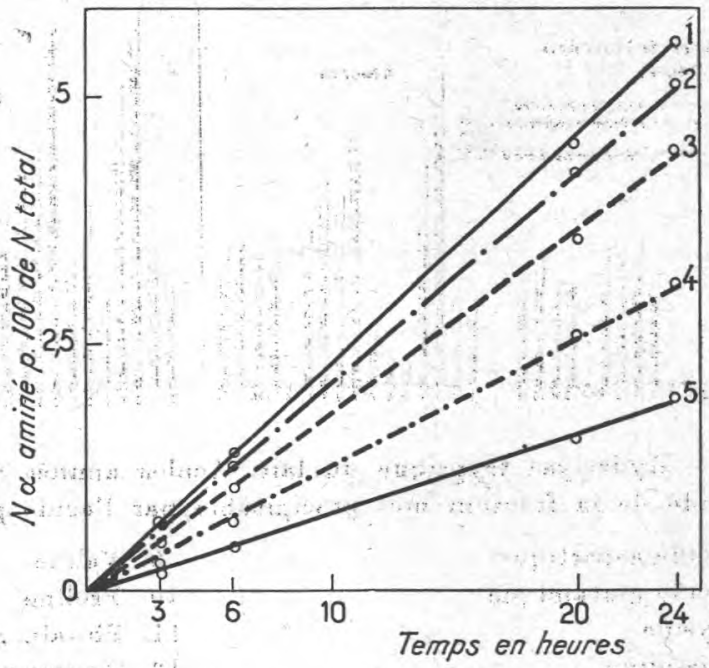

Fig. 4. Hydrolyse trypsique du lait soumis à différents traitements thermiques.

1. Cru.

2. Pasteurisé 30 sec à $90^{\circ} \mathrm{C}$.

3. Chauffé sur flamme $4 \mathrm{mn}$ à $122^{\circ} \mathrm{C}$.

4. Autoclavé (boîte) $12 \mathrm{mn}$ à $118^{\circ} \mathrm{C}$.

5. Autoclavé (bouteille) $12 \mathrm{mn}$ à $118^{\circ} \mathrm{C}$.

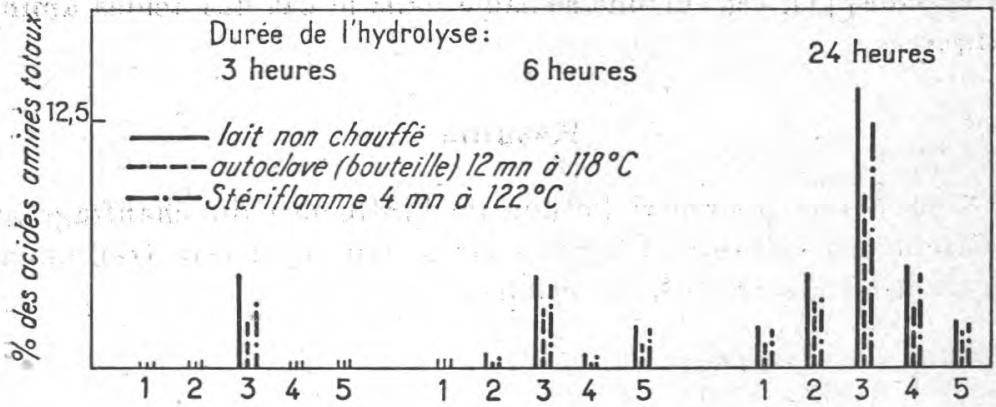

Fig. 5. - Acides aminés libérés lors de l'hydrolyse trypsique du lait.

1. Acide aspartique.

2. Acide glutamique.

3. Lysine.
4. Histidine.

5. Arginine. 


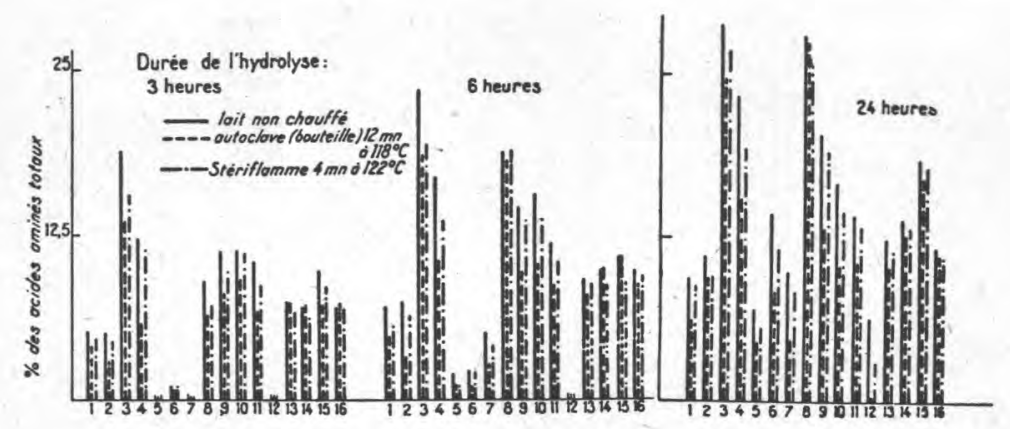

Fig. 6. - Hydrolyse trypsique du lait. Acides aminés libérés après hydrolyse acide de la fraction non précipitable par l'acide perchlorique.

1. Acide aspartique.

2. Acide glutamique.

3. Lysine.

4. Histidine.

5. Arginine.

6. Leucine.

7. Glycine.

8. $\alpha$-Alanine.
9. Valine.

10. Proline.

11. Ph. alanine.

12. Cystéine.

13. Méthionine.

14. Sérine.

15. Thréonine.

16. Tyrosine.

D'une façon générale, la stérilisation en boîte pendant $4 \mathrm{mi}$ nutes à $122^{\circ} \mathrm{C}$ retarde sensiblement moins la libération des acides aminés par la trypsine que la stérilisation en bouteille pendant 12 minutes à $118^{\circ} \mathrm{C}$.

Ce retard, qui se manifeste pour tout traitement thermique des caséines [1], est surtout sensible dans le cas des acides aminés basiques.

\section{Résumé}

Nous avons poursuivi l'étude de l'influence du chauffage sur l' $\alpha$-caséine en solution. L'action sur le lait de divers traitements thermiques industriels a été étudiée.

Solutions d'a-caséine.

Un chauffage de 40 minutes à $120^{\circ} \mathrm{C}$ a pour effet de doubler pratiquement la solubilité à $p \mathrm{H} 4,5$ de l' $\alpha$-caséine. Le chauffage entraîne également la dissimulation vis-à-vis du fluorodinitrobenzène d'une proportion notable des radicaux $\mathrm{NH}_{2}$ libres de la lysine et de l'arginine. 
La déphosphorylation enzymatique de l' $\alpha$-caséine conduit aux mêmes modifications de solubilité que la déphosphorylation par chauffage.

\section{Lait}

Solubilité, teneur en phosphore, "lysine disponible» selon Carpenter, allure de l'hydrolyse trypsique, vitesse de libération des acides aminés au cours de la protéolyse sont modifiées par la plupart des traitements thermiques industriels étudiés, à l'exception de la pasteurisation. Toutefois, les modifications produites par la stérilisation rapide par passage des boîtes sur flamme sont faibles.

En revanche, les traitements conventionnels de stérilisation modifient sensiblement les propriétés ci-dessus des caséines, et ce, d'autant plus que le chauffage appliqué est sévère.

\section{Summary}

We have continued the study of the influence of heating on $\alpha$-casein in solution. The action of various industrial heat treat. ments on milk has been studied.

\section{Solutions of $\alpha$-casein.}

Heating for 40 minutes at $120^{\circ} \mathrm{C}$ practically doubles the solubility of $\alpha$-casein at $p \mathrm{H} \mathrm{4,5}$. The heating also brings about masking towards fluorodinitrobenzene of a considerable proportion of free amino groups of lysine and arginine.

The enzymatic dephosphorylation of $\alpha$-casein produces the same modifications of solubility as dephosphorylation by heating.

\section{Milk.}

Solubility, phosphorus content, "available lysine» according to CARpenter, aspect of the tryptic hydrolysis, velocity of liberation of amino-acids in the course of proteolysis, are modified by the majority of the industrial heat treatments studied, except pasteurization. However, the modifications produced by rapid sterilization by passing the tins through a flame are small.

On the contrary, conventional treatments for sterilization modify the above-mentioned properties of caseins appreciably, and the more so the higher the heating applied. 


\section{Zusammenfassung}

Wir haben den Einfluss der Erwärmung auf das $\alpha$-Casein in Lösung weiter untersucht. Die Wirkung verschiedener thermischer Industriebehandlungen auf die Milch wurde untersucht.

\section{$\alpha$-Caseinlosungen.}

Eine Erwärmung von 40 Minuten bei $120^{\circ} \mathrm{C}$ bewirkt praktisch eine doppelte Löslichkeit des $\alpha$-Caseins bei $p$ H 4,5. Die Erwärmung ruft ebenso eine Verhüllung eines bedeutsamen Teiles der freien $\mathrm{NH}_{2}$-Radikale des Lysins und des Arginins gegen Fluordinitrobenzol hervor.

Die enzymatische Dephosphorylierung des $\alpha$-Caseins führt zu denselben Aenderungen wie die Dephosphorylierung durch Erwärmung.

\section{Milch.}

Die Löslichkeit, der Phosphorgehalt, das "verfügbare Lysin" nach CARPenter, der Lauf der Trypsinhydrolyse, die Freisetzungsgeschwindigkeit der Aminosäuren im Laufe der Proteolyse werden von der Mehrheit der untersuchten thermischen Industriebehandlungen, mit Ausnahme der Pasteurisation, geändert. Jedoch sind die, durch rasche Sterilisation durch Durchziehen der Dosen durch die Flamme, hervorgerufenen Aenderungen gering.

Hingegen ändern die üblichen Sterilisationsbehandlungen deutlich die oben erwähnten Eigenschaften der Caseine, desto mehr als die angewandte Erwärmung streng ist.

\section{BIBLIOGRAPHIE}

[1] M. Swirski, R. Allouf et H. Cheftel. Bull. Soc. Chim. Biol., 1961, 43, 909. - Le Lait, 1962, 42, 260 et 392.

[2] K. J. Carpenter et G. M. Ellinger. Biochem. J., 1955, 61, XI.

[3] D. Bruno et K. J. Carpenter. Biochem. J., 1957, 67, 13P.

[4] E. F. Mellon, A. H. Korn et S. R. Hoover. J. Am. Chem. Soc,. $1953,75,1675$.

[5] H. Wissman et H. Nitschmann. Helv. Chem. Acta, 1957, 40, 356.

[6] Ch. M. Iss. Thèse Biol., Univ. Florida, 1958.

[7] W. Manson. Nature, 1959, 184, 1393.

[8] R. C. Warner. J. Am. Chem. Soc., 1944, 66, 1725.

[9] J. J. Kelley. Thèse Biochim., Univ. Wisconsin, 1958.

[10] Ch. A. Zittle et E. Della Monica. Arch. Biochem., 1950, 26, 112.

[11] D. D. Van Slyke, R. T. Dillon, D. A. McFayden et P. Hamilton. J. Biol. Chem., 1941, 141, 627. 
[12] P. Hamilton et D. D. Van Slyke. J. Biol. Chem., 1943, 150, 231.

[13] W. G. Gordon, W. F. Semmett et M. Bender. J, Am. Chem. Soc., $1950,72,4282$.

[14] F. SANGer. Biochem. J., 1945, 39, 507.

[15] R. Monnier et L. Penasse, C. R. Acad. Sciences, 1950, 230, 1176.

[16] M. Beauvais, G. Thomas et H. Cheftel. Food Technology, April 1961, 15, 5 -9.

[17] T. A. Sundararajan et P. S. Sarma. Enzymologia, 1958, 20, 57.

[18] E. J. Bigwood et S. Payne Botha. Brit. J. Nutr., 1959, 13, 385.

\title{
REVUE
}

\section{LE LAIT DANS LE MONDE}

\author{
par \\ G. GENIN
}

Ingénieur E. P. C. I.

\section{ANGLETERRE}

\section{Nouveau dispositif pour l'analyse automatique du lait}

Le National Institute for Research in Dairying a mis au point un dispositif analytique qui permet de réduire les dépenses d'analyse du lait de 1 schilling à 3 pence par échantillon et de rendre automatique la détermination en 3 secondes de la teneur en graisse, en lactose et en protéine. Le nouvel appareillage, mis au point à Shinfield, utilise une technique d'absorption dans l'infrarouge et est surtout intéressant pour d'importants dépôts qui ont à contrôler de larges quantités de lait. Les fabricants de crème glacée trouveront également un avantage à utiliser cet appareil. Un prototype de ce dispositif a été récemment utilisé et a servi à procéder pendant six mois à l'analyse d'échantillons de lait fournis par des vaches à différentes périodes de lactation. La fabrication industrielle de ce dispositif va débuter prochainement.

\section{Nouveau mode de conditionnement du fromage à la bière}

Le fromage Ilchester, qui est constitué par un mélange de Cheddar et de bière de Worthington, de ciboulette et d'épices, est désormais vendu dans des pots en carton fabriqués par la Lily Cups and Containers Ltd. Ces cartons sont doublement imprégnés 